EPHOU 97-005

May 1997

\title{
Seiberg-Witten Theory of Rank Two Gauge Groups and Hypergeometric Series
}

\author{
Takahiro Masuda 1 , \\ Toru Sasaki i \\ and \\ Hisao Suzukif \\ Department of Physics, \\ Hokkaido University \\ Sapporo, Hokkaido 060 Japan
}

\begin{abstract}
In $S U(2)$ Seiberg-Witten theory, it is known that the dual pair of fields are expressed by hypergeometric functions. As for the theory with $S U(3)$ gauge symmetry without matters, it was shown that the dual pairs of fields can be expressed by means of the Appell function of type $F_{4}$. These expressions are convenient for analyzing analytic properties of fields. We investigate the relation between Seiberg-Witten theory of rank two gauge group without matters and hypergeometric series of two variables. It is shown that the relation between gauge theories and Appell functions can be observed for other classical gauge groups of rank two. For $B_{2}$ and $C_{2}$, the fields are written in terms of Appell functions of type $H_{5}$. For $D_{2}$, we can express fields by Appell functions of type $F_{4}$ which can be decomposed to two hypergeometric functions, corresponding to the fact $S O(4) \sim S U(2) \times S U(2)$. We also consider the integrable curve of type $C_{2}$ and show how the fields are expressed by Appell functions. However in the case of exceptional group $G_{2}$, our examination shows that they can be represented by hypergeometric series which does not correspond to the Appell functions.
\end{abstract}

\footnotetext{
*e-mail address: masuda@phys.hokudai.ac.jp

$\dagger$ e-mail address: sasaki@phys.hokudai.ac.jp

${ }^{\ddagger}$ e-mail address: hsuzuki@phys.hokudai.ac.jp
} 


\section{Introduction}

Many recent researches which have been originated to the work of Seiberg-Witten [1], are now clarifying the non-perturbative aspect of various supersymmetric gauge field theories through the duality symmetry. Seiberg and Witten gave the exact solution of the low energy effective theory with gauge group $S U(2)$ without matters, which provide a kind of understanding of the confinement through the monopole condensation. Moreover it was pointed out that there exist some special points in the moduli space of the extended theories constructed by introducing the matter fields and taking higher rank gauge symmetry, which realize the superconformal field theories [2, 3, 4]. Such possibilities of generalization have been studied extensively and the frameworks of the extended theories have been constructed elegantly [5, 6, 7, 8, 9, 10, 11, 12, 13].

The integrability of such known Seiberg-Witten type theories are insured by the relation to the integrable system [14], which shows that the hyperelliptic curve of the Seiberg-Witten model corresponds to the spectral curve of the periodic Toda lattice [15]. It is possible to construct the Seiberg-Witten model with the classical gauge group including some cases of the exceptional gauge group [16, 17, 18], and also the model which has no corresponding gauge group, all by using suitable spectral curve of integrable systems [15]. Recently it was pointed out that equivalent curves are obtained by the fibrations of ALE spaces of type II superstring theories conpactified on Calabi-Yau threefolds [19, 20, 21, 22, although such equivalence is not manifest but physical in the case of the exceptional group [18, 23.

As for the explicit evaluation of the theory, various methods to obtain the physical information in such Seiberg-Witten models have been investigated. One major way is to use the concrete expression of dual Higgs pairs obtained by solving the Picard-Fuchs equation [24] or evaluating the integral representation [25, 26], to get the low energy

effective action. There are also direct calculations for the prepotential [27] which have been developed in relating the soliton theory [28 in the weak coupling region. These result have been almost consistently checked by the direct instanton calculus in the weak coupling region [29], especially have shown good coincidence in the case without matters in the classical gauge group [30]. The evaluations for the prepotential have been carried out even in the strong coupling region [31]. In the case of $S U(2)$, besides the 
prepotential, the BPS spectrum have been analyzed in both regions by means of global quantum symmetry [32]. Soft breaking of $N=2$ supersymmetry with massless or massive hypermultiplets has been investigated due to the strong coupling analysis [33.

Although physical information can be given by the prepotential, in this article we concentrate on obtaining analytic expressions of dual Higgs pairs exactly to investigate their analytic properties. It is known that they should be represented by hypergeometric series in the classical gauge group [25, 26], and obtained order by order recursively by analyzing the Picard-Fuchs equations [12, 34, 35, 36, 30]. We choose an alternative approach to evaluate the integral representation which has been investigated among our previous works [26, 37]. In the case of $S U(n)$ and $S O(2 n)$ theory, results obtained by this method are expressed by using hypergeometric series [26], however this representation does not seem to be convenient to see analytic behaviors for generic region of the branch. In other words, we have to perform the expansion in various regions where we can not obtain the result expanded in terms of the hypergeometric series. However in the case of $S U(3)$ [12], the analytic property is shown to be apparent because the solution can be written by Appell functions. These functions are second order hypergeometric series in two variables, and can be represented by using the simple hypergeometric functions in terms of each variable, so that we can obtain the expression close to the boundary to the convergence region. Therefore the rank two gauge groups are expected to be special cases where solutions can be represented by analogous hypergeometric functions in a uniform way so that their analytic properties can be seen manifestly.

Our aim in this article is to clarify the relation between hypergeometric series and $N=2$ supersymmetric Yang-Mills theories with rank two gauge group both by use of explicit evaluations and by the Picard-Fuchs equations. As the result, we will show that the dual pairs of fields are represented by Appell functions for the classical gauge groups of rank two. In the case of exceptional group $G_{2}$, the solution of the dual pairs of fields have been obtained not in the form of hypergeometric series in [38]. In this article we will find that we can obtain the expression in the form of hypergeometric series. However they turn out to be the third order hypergeometric series, which means that they are not within the Appell system in this case. Above solutions obtained by explicit evaluations can satisfy the Picard-Fuchs equations of these theories as we will see in this article.

This article is organized as follows. In section two, we will review the theory with gauge group $S U(3)$ and will show how to obtain the result by Klemm et al. [12 by explicit 
evaluations of the integral. In section three, we will calculate in the case of $S O(5)$ and $S p(4)$. In section four we will deal with the theory with $S O(4)$, so that we see that results reflect the fact $S O(4) \sim S U(2) \times S U(2)$. In section five we will consider the integrable system $C_{2}$ whose counterpart gauge group is not known now. From sect. 3 to sect. 5, the results can be represented by suitable Appell functions with various parameterizations. In section six, we will deal with exceptional group $G_{2}$ to show that the solution can be represented by the generalized hypergeometric function of order 3 in terms of a special parameterization of the moduli parameter. Last section will be devoted some discussion.

\section{Review of $S U(3)$ gauge theory}

To begin with, we review $S U(3)$ gauge theory whose dual pairs were previously evaluated exactly by Klemm, Lerche and Theisen [12] by means of solving the Picard-Fuchs equation directly. We are going to evaluate dual pairs of $S U(3)$ gauge theory by integrating meromorphic differential $\lambda$ explicitly in the weak coupling region to see that we can recover the exact solutions. Hyperelliptic curve and meromorphic differential $\lambda$ of $S U(3)$ gauge theory are given by

$$
\begin{gathered}
y^{2}=W^{2}-\Lambda^{6}, W=x^{3}-u x-v, \\
\lambda=\frac{x W^{\prime}}{y} d x .
\end{gathered}
$$

Higgs field $a_{i}$ and its dual $a_{D}^{i}(i=1,2,3)$ are given by

$$
a_{i}=\oint_{\alpha_{i}} \lambda, a_{D}^{i}=\oint_{\beta_{i}} \lambda
$$

where $\sum_{i=1}^{3} a_{i}=0$, and $\alpha_{i}, \beta_{i}(i=1,2,3)$ are basis of homology cycle. In the weak coupling region $(\Lambda \sim 0)$, we expand $1 / y$ of $\lambda$ with small $\Lambda$ [26, 37]

$$
\lambda=d x \int_{-i \infty}^{+i \infty} \frac{d s}{2 \pi i} \frac{\Gamma(-s) \Gamma\left(\frac{1}{2}+s\right)}{\Gamma\left(\frac{1}{2}\right) 2 s}\left(-\Lambda^{6}\right)^{s} W^{-2 s},
$$

where we introduce $s$ integral which picks up poles at $s=0,1,2, \cdots$, in place of summation, and integrate by parts. The detailed calculation is given in Appendix A. We first perform the contour integral along $\alpha_{i}$ and $\beta_{i}$ cycle, next evaluate s integral. In the weak 
coupling region the integral of $\lambda$ along $\alpha_{i}$ cycle picks up simple poles of (2.4). In the region $u \sim \infty$, we take $\alpha_{1}\left(\alpha_{2}\right)$ cycle to enclose two point $x \sim \sqrt{u}(-\sqrt{u})$ and $x \sim v / u \sim 0$, so as to be same basis taken by Klemm et al. [12]. Moreover this contour integration can be carried out by line integration from $x=0$ to $x=\sqrt{u}(-\sqrt{u})$ supplemented by $\sin 2 s \pi / \pi$. Similarly the integration along $\beta_{i}$ cycle can be replaced to this line integration without multiplied by $\sin 2 \pi s$ because this contour initially intersects the blanch cut. Since the reduction of two different roots of the curve (2.1) to one classical root caused by $\Lambda \rightarrow 0$, provides an excessive contribution $\alpha_{i} / 2$ to $\beta_{i}$, we have to subtract this. Therefore [26, 37]

$$
\begin{aligned}
& a_{1}=\int_{0}^{\sqrt{u}} \lambda \frac{\sin 2 \pi s}{\pi}, \quad a_{D}^{1}=\frac{1}{2 \pi i} \int_{0}^{\sqrt{u}} \lambda-\frac{1}{2} a_{1}, \\
& a_{2}=\int_{0}^{-\sqrt{u}} \lambda \frac{\sin 2 \pi s}{\pi}, \quad a_{D}^{2}=\frac{1}{2 \pi i} \int_{0}^{-\sqrt{u}} \lambda-\frac{1}{2} a_{2},
\end{aligned}
$$

To be concrete, we expand $W^{-2 s}$ by $1 /\left(x^{3}-u x\right)$, and integrate $\lambda$ by $x$ from $x=0$ to $x=\sqrt{u}(x=-\sqrt{u})$ and evaluate $s$ integration. After some arrangement we obtain

$$
\begin{aligned}
a_{1}= & \sqrt{u} F_{4}\left(-\frac{1}{6}, \frac{1}{6}, 1, \frac{1}{2} ; \frac{27 \Lambda^{6}}{4 u^{3}}, \frac{27 v^{2}}{4 u^{3}}\right)+\frac{v}{2 u} F_{4}\left(\frac{1}{3}, \frac{2}{3}, 1, \frac{3}{2} ; \frac{27 \Lambda^{6}}{4 u^{3}}, \frac{27 v^{2}}{4 u^{3}}\right) \\
a_{D}^{1}= & \frac{1}{2 \pi i} \sqrt{u} \sum_{m, n} \frac{\left(-\frac{1}{6}\right)_{m+n}\left(\frac{1}{6}\right)_{m+n}}{m ! n !(1)_{m}\left(\frac{1}{2}\right)_{n}}\left(\frac{27 \Lambda^{6}}{4 u^{3}}\right)^{m}\left(\frac{27 v^{2}}{4 u^{3}}\right)^{n} \\
& \times\left[-2 \psi(m+1)+\psi\left(-\frac{1}{6}+m+n\right)+\psi\left(\frac{1}{6}+m+n\right)+\log \left(-\frac{27 \Lambda^{6}}{4 u^{3}}\right)\right] \\
+ & \frac{1}{2 \pi i} \frac{v}{2 u} \sum_{m, n} \frac{\left(\frac{1}{3}\right)_{m+n}\left(\frac{2}{3}\right)_{m+n}}{m ! n !(1)_{m}\left(\frac{3}{2}\right)_{n}}\left(\frac{27 \Lambda^{6}}{4 u^{3}}\right)^{m}\left(\frac{27 v^{2}}{4 u^{3}}\right)^{n} \\
& \times\left[-2 \psi(m+1)+\psi\left(\frac{1}{3}+m+n\right)+\psi\left(\frac{2}{3}+m+n\right)+\log \left(-\frac{27 \Lambda^{6}}{4 u^{3}}\right)\right] \\
a_{2}= & \sqrt{u} F_{4}\left(-\frac{1}{6}, \frac{1}{6}, 1, \frac{1}{2} ; \frac{27 \Lambda^{6}}{4 u^{3}}, \frac{27 v^{2}}{4 u^{3}}\right)+\frac{v}{2 u} F_{4}\left(\frac{1}{3}, \frac{2}{3}, 1, \frac{3}{2} ; \frac{27 \Lambda^{6}}{4 u^{3}}, \frac{27 v^{2}}{4 u^{3}}\right) \\
a_{D}^{2}= & \frac{1}{2 \pi i} \sqrt{u} \sum_{m, n} \frac{\left(-\frac{1}{6}\right)_{m+n}\left(\frac{1}{6}\right)_{m+n}}{m ! n !(1)_{m}\left(\frac{1}{2}\right)_{n}}\left(\frac{27 \Lambda^{6}}{4 u^{3}}\right)^{m}\left(\frac{27 v^{2}}{4 u^{3}}\right)^{n} \\
& \times\left[-2 \psi(m+1)+\psi\left(-\frac{1}{6}+m+n\right)+\psi\left(\frac{1}{6}+m+n\right)+\log \left(-\frac{27 \Lambda^{6}}{4 u^{3}}\right)\right] \\
+ & \frac{1}{2 \pi i} \frac{v}{2 u} \sum_{m, n} \frac{\left(\frac{1}{3}\right)_{m+n}\left(\frac{2}{3}\right)_{m+n}}{m ! n !(1)_{m}\left(\frac{3}{2}\right)_{n}}\left(\frac{27 \Lambda^{6}}{4 u^{3}}\right)^{m}\left(\frac{27 v^{2}}{4 u^{3}}\right)^{n} \\
& \times\left[-2 \psi(m+1)+\psi\left(\frac{1}{3}+m+n\right)+\psi\left(\frac{2}{3}+m+n\right)+\log \left(-\frac{27 \Lambda^{6}}{4 u^{3}}\right)\right],
\end{aligned}
$$


where $(\alpha)_{m}=\Gamma(a+m) / \Gamma(a), \psi(x)=\Gamma^{\prime}(x) / \Gamma(x)$ and defining expression of $F_{4}$ function is given by

$$
F_{4}(\alpha, \beta, \gamma, \delta ; x, y)=\sum_{m, n} \frac{(\alpha)_{m+n}(\beta)_{m+n}}{m ! n !(\gamma)_{m}(\delta)_{n}} x^{m} y^{n}
$$

This is the Appell $F_{4}$ function which are order two hypergeometric series in terms of each variables [39]. The solutions (2.7)-(2.10) are equal to the results obtained by Klemm et al. 12 up to over all sign of $a_{2}, a_{D}^{2}$.

Analytic property of $F_{4}$ function is apparent because this is a natural extension of Gaussian sum in two variables and is expressed by using hypergeometric functions of $x$ as

$$
F_{4}(\alpha, \beta, \gamma, \delta ; x, y)=\sum_{n} \frac{(\alpha)_{n}(\beta)_{n} y^{n}}{(\delta)_{n} n !} F(\alpha+n, \beta+n, \gamma, x)
$$

where the hypergeometric function $F(a, b, c ; z)$ is defined by

$$
F(a, b, c ; z)=\sum_{n} \frac{(a)_{n}(b)_{n}}{(c)_{n} n !} z^{n}
$$

and similar manipulation can be carried out in terms of $y$. Therefore it is easy to know the behavior near the boundary of the convergence region. That is, an application of the analytic continuation formula of the hypergeometric function in terms of $x$ or $y$ to (2.12) yields the expression in some region we want. In this case we should pay a little bit attention to deal with logarithmic functions because the results of the following manipulations generally depend on the choice of the branch of the logarithmic function. Let us see how the solutions are transformed to other Appell functions in various regions by these analytic properties. If we take the branch for large $v$ in the weak coupling region, by taking inversion of the variable $27 v^{2} / 4 u^{3}$ of the expression (2.7)-(2.10), we can give the solutions in the form of $F_{4}$ function, to be precise, $F_{4}\left(-1 / 6,1 / 3 ; 2 / 3,1 ; 4 u^{3} / 27 v^{2}, \Lambda^{6} / v^{2}\right)$ and its independent solutions. Furthermore this $F_{4}$ function can be written by $H_{4}$ function 40

$$
H_{4}(a, b, c, 2 b ; z, w)=\left(1-\frac{1}{2} w\right)^{-a} F_{4}\left(\frac{1}{2} a, \frac{1}{2} a+\frac{1}{2}, c, b+\frac{1}{2} ; \frac{16 z}{(2-w)^{2}}, \frac{w^{2}}{(2-w)^{2}}\right),(2 .
$$

where $a=-1 / 3, b=1 / 2, c=2 / 3, z=\left( \pm v+\Lambda^{3}\right)^{2} u^{3} / 108, w=2 \Lambda^{3} /\left( \pm v+\Lambda^{3}\right)$, and $H_{4}$ is another Appell function defined as

$$
H_{4}(\alpha, \beta, \gamma, \delta ; z, w)=\sum_{m, n} \frac{(\alpha)_{2 m+n}(\beta)_{n}}{(\gamma)_{m}(\delta)_{n} m ! n !} z^{m} w^{n} .
$$


Notice that this function also can be written by the hypergeometric function with $z$ or $w$. Although the formula (2.14) looks like the transformation about both two variables, only one variable $w$ is in fact transformed by using the quadratic transformation of the hypergeometric function. Similarly it is possible to transform the solutions by using well-known identities of the hypergeometric function, and in some cases they can be represented by Appell functions as the result of the transformation [39, 40].

The most interesting region on the physical point of view is around $\mathbf{Z}_{3}$ point $u=$ $0, v= \pm \Lambda^{3}$ where the theory looks like the superconformal field theory [2]. This point lies on the boundary of the convergence region of the expression $F_{4}$ for large $v$, and already have appeared in the variables of $H_{4}$. Therefore the solutions around this point can be given by analytic continuation for $\Lambda^{6} / v^{2} \sim 1$ from $F_{4}$, or by the inversion of $w=2 \Lambda^{3} /\left( \pm v+\Lambda^{3}\right)$ of $H_{4}$. After some arrangements for the results to be simple forms, the solutions can be written by Appell $H_{7}$ functions. For detailed calculations, see ref. [26]. In this case, these analytic expressions show following properties; the logarithmic part of the solutions disappear after this analytic continuation and Higgs field $a_{i}$ and its dual $a_{D}^{i}$ are on the equal standing up to overall constant, and their leading dependence of moduli parameter becomes of fractional power [26]. These properties indicate that the theory realize superconformal invariance on this point as a nontrivial IR fixed point [2].

Next let us review how these four solutions satisfy the Picard-Fuchs equations of this theory. The Picard-Fuchs equations for $\Pi=\oint \lambda$ are given by $\mathcal{L}_{i} \Pi=0(i=0,1)$ where $\mathcal{L}_{i}$ is written by

$$
\begin{aligned}
& \mathcal{L}_{0}=-3 \partial_{u}^{2}+u \partial_{v}^{2}, \\
& \mathcal{L}_{1}=4 u^{2} \partial_{u}^{2}-9\left(\Lambda^{6}-v^{2}\right) \partial_{v}^{2}+12 u v \partial_{u} \partial_{v}+3 v \partial_{v}+1 .
\end{aligned}
$$

By changing variables directly as $x=\frac{27 \Lambda^{6}}{4 u^{3}}, y=\frac{27 v^{2}}{4 u^{3}}$ and setting $\Pi=\sqrt{u} F$, we can write the differential equation for $F$ in the form as $\tilde{\mathcal{L}}_{i} F=0(i=0,1)$ where $\tilde{\mathcal{L}}_{i}$ is given by

$$
\begin{aligned}
& \tilde{\mathcal{L}}_{0}=-x^{2} \partial_{x}^{2}-2 x y \partial_{x} \partial_{y}+y(1-y) \partial_{y}^{2}-x \partial_{x}+\left(\frac{1}{2}-y\right) \partial_{y}+\frac{1}{36}, \\
& \tilde{\mathcal{L}}_{1}=x(1-x) \partial_{x}^{2}-2 x y \partial_{x} \partial_{y}-y^{2} \partial_{y}^{2}+(1-x) \partial_{x}-y \partial_{y}+\frac{1}{36} .
\end{aligned}
$$

This is Appell $F_{4}$ system constructed from the definition [39], whose independent four solutions turn out to be just the solutions derived in this section by linear combinations. 


\section{$3 B_{2}$ and $C_{2}$ gauge theory}

In this section we perform the evaluation in the case of $B_{2}(S O(5))$ gauge theory at first, and $C_{2}(S p(4))$ in the last of this section whose results can be given by a reparameterization of the results of $B_{2}$ case. Hyperelliptic curve and meromorphic differential $\lambda$ of $B_{2}$ theory are given by

$$
\begin{gathered}
y^{2}=W(x)^{2}-\Lambda^{6} x^{2}, \quad W(x)=x^{4}-u x^{2}+v \\
\lambda=\frac{x W^{\prime}(x)-W(x)}{y} d x \\
=d x \int_{-i \infty}^{i \infty} \frac{d s}{2 \pi i} \frac{\Gamma\left(\frac{1}{2}+s\right) \Gamma(-s)(-1)^{s}\left(\Lambda^{6}\right)^{6}}{\Gamma\left(\frac{1}{2}\right) 2 s}\left(\frac{x^{2}}{W(x)^{2}}\right)^{s},
\end{gathered}
$$

where we expand $\lambda$ in the weak coupling region, and introduce $s$ integral, and integrate by parts. In this case, we set $\beta_{i}$ cycle to intersect $\alpha_{i}$ cycle and its copy $\alpha_{i}^{\prime}$ cycle both, because curve (3.1) is even function in terms of $x$. In the region $u \sim \infty, a_{1}$ consists of the contribution from $x^{2} \sim v / u$, and $a_{2}$ consists of from $x^{2} \sim u$. Therefore $a_{1}$ can be obtained by expanding $\lambda$ with respect to $1 /\left(u x^{2}-v\right)$ and performing $x$ integral of $\lambda$ from $x=-\sqrt{v / u}$ to $x=\sqrt{v / u}$ divide by 2 , and multiplied by $\sin 2 \pi s / \pi$ so as to pick up the simple pole to evaluate integration along $\alpha_{1}$. Corresponding $a_{D}^{1}$ is obtained by integrating without multiplied by $\sin 2 \pi s$ in place of integration along $\beta_{1}$, by evaluating double poles and by subtracting excessive contributions. The result can be written by

$$
\begin{aligned}
a_{1}= & \sqrt{\frac{v}{u}} \sum_{m, n} \frac{\left(\frac{1}{2}\right)_{n+2 m}\left(-\frac{1}{2}\right)_{n-m}}{n ! m !(1)_{n}}\left(\frac{v}{u^{2}}\right)^{m}\left(-\frac{\Lambda^{6}}{4 u v}\right)^{n} \\
= & \sqrt{\frac{v}{u}} H_{5}\left(\frac{1}{2},-\frac{1}{2}, 1 ; \frac{v}{u^{2}},-\frac{\Lambda^{6}}{4 u v}\right), \\
a_{D}^{1}= & \frac{1}{2 \pi i} \sqrt{\frac{v}{u}} \sum_{m, n} \frac{\left(\frac{1}{2}\right)_{n+2 m}\left(-\frac{1}{2}\right)_{n-m}}{n ! m !(1)_{n}}\left(\frac{v}{u^{2}}\right)^{m}\left(-\frac{\Lambda^{6}}{4 u v}\right)^{n} \\
& \times\left[-2 \psi(n+1)+\psi\left(n+2 m+\frac{1}{2}\right)+\psi\left(-\frac{1}{2}+n-m\right)+\log \left(-\frac{\Lambda^{6}}{4 u v}\right)\right] .
\end{aligned}
$$

These are Appell $H_{5}$ functions which are order two hypergeometric series in terms of each variables defined by 39

$$
H_{5}(a, b, c ; x, y)=\sum_{m, n} \frac{(a)_{n+2 m}(b)_{n-m}}{(c)_{n} m ! n !} x^{m} y^{n},
$$


which can be represented by using hypergeometric functions in terms of $-x$ and $y$ respectively. Contrast to $F_{4}$ function in the case of $S U(3)$, other solutions are not necessary represented by order two hypergeometric series as we will see in the following. By similar evaluations, we can get the expression for $a_{2}$ and $a_{D}^{2}$ by expanding $\lambda$ with $1 /\left(u x^{2}-v\right)$ and by replacing lower and upper value of the line integral to $x=-\sqrt{u}$ and $x=\sqrt{u}$ respectively

$$
\begin{aligned}
a_{2}= & u^{\frac{1}{2}} \sum_{m, n} \frac{\left(-\frac{1}{2}\right)_{3 m+2 n}}{\left(\frac{1}{2}\right)_{m+n} m ! m ! n !}\left(\frac{\Lambda^{6}}{4 u^{3}}\right)^{m}\left(-\frac{v}{u^{2}}\right)^{n} \\
a_{D}^{2}= & \frac{u^{\frac{1}{2}}}{2 \pi i} \sum_{m, n} \frac{\left(-\frac{1}{2}\right)_{3 m+2 n}}{\left(\frac{1}{2}\right)_{m+n} m ! m ! n !}\left(\frac{\Lambda^{6}}{4 u^{3}}\right)^{m}\left(-\frac{v}{u^{2}}\right)^{n} \\
& \times\left[2 \psi(m+1)-\psi\left(\frac{1}{2}+m+n\right)+3 \psi\left(-\frac{1}{2}+3 m+2 n\right)+\log \left(-\frac{\Lambda^{6}}{4 u^{3}}\right)\right] .
\end{aligned}
$$

Thus we see that these solutions are of order three in terms of one variable. Although analytic property of the expression (3.6) and (3.7) are less manifest, we can see the behavior in various regions by using analytic property of the $H_{5}$ function (3.4) and (3.5).

Next let us see how these four solutions can satisfy the Picard-Fuchs equations of this theory. Picard-Fuchs equations for $\Pi=\oint \lambda$ are given by $\mathcal{L}_{i} \Pi=0(i=0,1)$ where $\mathcal{L}_{i}$ is written by

$$
\begin{aligned}
& \mathcal{L}_{0}=3 \partial_{u}^{2}+u \partial_{u} \partial_{v}-v \partial_{v}^{2}, \\
& \mathcal{L}_{1}=4 u^{2} \partial_{u}^{2}-\left(9 \Lambda^{6}-16 u v\right) \partial_{u} \partial_{v}+16 v^{2} \partial_{v}^{2}+8 v \partial_{v}+1 .
\end{aligned}
$$

By changing variables directly as $x=\frac{v}{u^{2}}, y=-\frac{\Lambda^{6}}{4 u v}$ and setting $\Pi=\sqrt{\frac{v}{u}} H$, we can write the differential equations for $H$ in the form as $\tilde{\mathcal{L}}_{i} H=0(i=0,1)$ where $\tilde{\mathcal{L}}_{i}$ are given by

$$
\begin{aligned}
& \tilde{\mathcal{L}}_{0}=x(1+4 x) \partial_{x}^{2}-y(1-4 x) \partial_{x} \partial_{y}+y^{2} \partial_{y}^{2}+\left(\frac{1}{2}+8 x\right) \partial_{x}+3 y \partial_{y}+\frac{3}{4}, \\
& \tilde{\mathcal{L}}_{1}=2 x^{2} \partial_{x}^{2}-x y \partial_{x} \partial_{y}+y(1-y) \partial_{y}^{2}+\frac{7}{2} x \partial_{x}+(1-y) \partial_{y}+\frac{1}{4} .
\end{aligned}
$$

These are the differential equations of Appell function $H_{5}$ constructed from the definition [39], whose independent four solutions are just our solutions obtained in this section.

In the rest of this section we consider the case with gauge group $C_{2}$. It is known that correct holomorphic one-form and meromorphic differential can be obtained from spectral curve of the integral system $C_{2}^{\vee}$ defined by [15]

$$
f=\left(z-\frac{\mu}{z}\right)^{2}+x^{2} W(x)=0, \quad W(x)=x^{4}-u x^{2}+v .
$$


Meromorphic differential $\lambda$ which we use to get dual Higgs pairs in the gauge theory is given from this curve by

$$
\lambda=x \frac{d z}{z}
$$

Regarding $\partial_{v} \lambda$ as $d x / y$, this manipulation corresponds to take hyperelliptic curve as

$$
y^{2}=x^{4} W(x)^{2}-\Lambda^{6} x^{2} W(x),
$$

where we set $\mu \propto \Lambda^{6}$. Compared to curve (3.1) this curve does not seem to reflect the property of group theory $S O(5) \sim S p(4)$. As was pointed out by Ito and Sasakura [30], the result of $C_{2}$ is obtained from results of $B_{2}$ by shifting moduli parameters as

$$
u^{C}=u^{B}, \quad v^{C}=v^{B}+\frac{1}{4}\left(u^{B}\right)^{2} .
$$

This is observed by noticing that in this parameterization the Picard-Fuchs equations of both theories coincide [30]. Thus the solution of $C_{2}$ theory are given by Appell $H_{5}$ system with variables constructed by $u^{C}, v^{C}$. This is also observed by explicit evaluation in a same way as in the case of $B_{2}$ by using curve (3.14), and analytic continuation with respect to this change of parameterization. For example $a_{1}$ can be written as

$$
\begin{aligned}
a_{1} & =\sum_{m, n} \sqrt{\frac{v^{C}}{u^{C}}} \frac{\left(\frac{1}{2}\right)_{m}\left(\frac{1}{2}\right)_{2 n-m}}{\left(\frac{3}{2}\right)_{n-2 m} m ! m ! n !}\left(\frac{-\Lambda_{C}^{6} u^{C}}{v^{C 2}}\right)^{m}\left(\frac{v^{C}}{u^{C 2}}\right)^{n} \\
& =\sqrt{\frac{v^{C}}{u^{C}}} \sum_{m} \frac{(-1)^{m}}{\left(\frac{3}{2}\right)_{-2 m} m ! m !}\left(-\frac{\Lambda_{C}^{6} u^{C}}{v^{C 2}}\right)^{m} F\left(-\frac{m}{2}+\frac{1}{4},-\frac{m}{2}+\frac{3}{4},-2 m+\frac{3}{2} ; \frac{4 v^{C}}{u^{C 2}}\right)(3
\end{aligned}
$$

This function is not the Appell function, and similarly other three independent solutions are not within Appell functions. However by analytic continuation of the hypergeometric function

$$
\begin{aligned}
\frac{1}{\Gamma(c)} F(a, b, c, z)= & \frac{\Gamma(a+b-c)}{\Gamma(a) \Gamma(b)}(1-z)^{c-a-b} F(c-a, c-b, c-a-b+1 ; 1-z) \\
& +\frac{\Gamma(c-a-b)}{\Gamma(c-a) \Gamma(c-b)} F(a, b, a+b-c+1 ; 1-z),
\end{aligned}
$$

and by use of the identity

$$
F(a, b, c ; z)=(1-z)^{c-a-b} F(c-a, c-b, c ; z),
$$


it is possible to get the expression in terms $u^{B}$ and $v^{B}$ as a linear combination of the solutions of $B_{2}$

$$
\begin{aligned}
a_{1}=\frac{1}{2 \sqrt{2 \pi}} \sqrt{\frac{v^{B}}{u^{B}}} & H_{5}\left(\frac{1}{2},-\frac{1}{2}, 1 ; \frac{v^{B}}{u^{B 2}},-\frac{\Lambda_{B}^{6}}{4 u^{B} v^{B}}\right) \\
& +\frac{1}{\sqrt{2 \pi}} \sqrt{u^{B}} \sum_{m, n} \frac{\left(-\frac{1}{2}\right)_{3 m+2 n}}{\left(\frac{1}{2}\right)_{m+n} m ! m ! n !}\left(\frac{\Lambda_{B}^{6}}{4 u^{B 3}}\right)^{m}\left(-\frac{v^{B}}{u^{B 2}}\right)^{n}
\end{aligned}
$$

where we set $\Lambda_{B}^{6}=-8 \Lambda_{C}^{6}$. Similarly we can evaluate to give $a_{2}, a_{D}^{1}$ and $a_{D}^{2}$ as some linear combinations of the result of $B_{2}$. Alternatively we can also verify by evaluations in a similar way initially by using this shifted parameterization as

$$
\widetilde{W}(x)=x^{4}-u^{B} x^{2}+\frac{1}{4} u^{B 2}+v^{B} .
$$

\section{$4 \quad S O(4)$ theory}

In this section we deal with the $S O(4)$ theory whose hyperelliptic curve is given by

$$
y^{2}=W(x)^{2}-\Lambda^{4} x^{4}, \quad W(x)=x^{4}-u x^{2}+v
$$

In relating to the change of the curve from the $B_{2}$ case, meromorphic differential $\lambda$ is varied slightly as

$$
\begin{aligned}
\lambda & =\frac{x W^{\prime}(x)-2 W(x)}{y} d x \\
& =d x \int_{-i \infty}^{i \infty} \frac{d s}{2 \pi i} \frac{\Gamma\left(\frac{1}{2}+s\right) \Gamma(-s)\left(-\Lambda^{4}\right)^{s}}{\Gamma\left(\frac{1}{2}\right) 2 s}\left(\frac{x^{4}}{W(x)^{2}}\right)^{s} .
\end{aligned}
$$

Since the modification from the $B_{2}$ theory is only powers of $x$ in the instanton term, we are able to evaluate the integral in the region $\Lambda \sim 0, u \sim \infty$ in a same way as in the $B_{2}$ case. The result can be expressed by

$$
\begin{aligned}
a_{1} & =\sqrt{u} F_{4}\left(-\frac{1}{4}, \frac{1}{4}, \frac{1}{2}, 1 ; \frac{4 v}{u^{2}}, \frac{\Lambda^{4}}{u^{2}}\right) \\
a_{2} & =\sqrt{\frac{v}{u}} F_{4}\left(\frac{1}{4}, \frac{3}{4}, \frac{3}{2}, 1 ; \frac{4 v}{u^{2}}, \frac{\Lambda^{4}}{u^{2}}\right) \\
a_{D}^{1} & =\frac{\sqrt{u}}{2 \pi i} \sum_{m, n} \frac{\left(-\left(\frac{1}{4}\right)_{m+n}\left(\frac{1}{4}\right)_{m+n}\right.}{\left(\frac{1}{2}\right)_{m}(1)_{n} m ! n !}\left(\frac{4 v}{u^{2}}\right)^{m}\left(\frac{\Lambda^{4}}{u^{2}}\right)^{n}
\end{aligned}
$$




$$
\begin{aligned}
& \times\left[-2 \psi(n+1)+\psi\left(n+m-\frac{1}{4}\right)+\psi\left(n+m+\frac{1}{4}\right)+\log \left(\frac{\Lambda^{4}}{u^{2}}\right)\right], \\
a_{D}^{2}= & \frac{1}{2 \pi i} \sqrt{\frac{v}{u}} \sum_{m, n} \frac{\left(\frac{1}{4}\right)_{m+n}\left(\frac{3}{4}\right)_{m+n}}{\left(\frac{1}{2}\right)_{m}(1)_{n} m ! n !}\left(\frac{4 v}{u^{2}}\right)^{m}\left(\frac{\Lambda^{4}}{u^{2}}\right) \\
& \times\left[-2 \psi(n+1)+\psi\left(\frac{1}{4}+m+n\right)+\psi\left(\frac{3}{4}+m+n\right)+\log \left(\frac{\Lambda^{4}}{u^{2}}\right)\right] .
\end{aligned}
$$

Again these solutions are written by Appell $F_{4}$ functions. It is interesting to see how this result reflect the fact $S O(4) \sim S U(2) \times S U(2)$. To this end, we use following identities to relate $F_{4}$ function to the usual hypergeometric function

$$
\begin{aligned}
F_{4}\left(\alpha, \alpha+\frac{1}{2}, \frac{1}{2}, \gamma ; x, y\right)= & \frac{(1+\sqrt{x})^{-2 \alpha}}{2} F\left(\alpha, \alpha+\frac{1}{2}, \gamma ; \frac{y}{(1+\sqrt{x})^{2}}\right) \\
& +\frac{(1-\sqrt{x})^{-2 \alpha}}{2} F\left(\alpha, \alpha+\frac{1}{2}, \gamma ; \frac{y}{(1-\sqrt{x})^{2}}\right), \\
F_{4}\left(\alpha+\frac{1}{2}, \alpha+1, \frac{3}{2}, \gamma ; x, y\right)= & \frac{-x^{-\frac{1}{2}}}{4 \alpha}\left\{(1+\sqrt{x})^{-2 \alpha} F\left(\alpha, \alpha+\frac{1}{2}, \gamma ; \frac{y}{(1+\sqrt{x})^{2}}\right)\right. \\
& \left.-(1-\sqrt{x})^{-2 \alpha} F\left(\alpha, \alpha+\frac{1}{2}, \gamma ; \frac{y}{(1-\sqrt{x})^{2}}\right)\right\} .
\end{aligned}
$$

The first formula is cited in Ref. [41], and second formula has been obtained in Ref. [42. Applying these identities with $\alpha=-1 / 4, a_{1}$ can be expressed by using two same hypergeometric functions of different variables as

$$
\begin{aligned}
a_{1}= & \frac{1}{2}(u+\sqrt{4 v})^{\frac{1}{2}} F\left(\frac{1}{4},-\frac{1}{4}, 1 ; \frac{\Lambda^{4}}{(u+\sqrt{4 v})^{2}}\right) \\
& +\frac{1}{2}(u-\sqrt{4 v})^{\frac{1}{2}} F\left(\frac{1}{4},-\frac{1}{4}, 1 ; \frac{\Lambda^{4}}{(u-\sqrt{4 v})^{2}}\right),
\end{aligned}
$$

where $a_{2}$ can be given by changing sign of second term. Noticing that in the case of $S U(2)$ theory Higgs field $a$ is written by [1]

$$
a=\sqrt{u} F\left(\frac{1}{4},-\frac{1}{4}, 1 ; \frac{\Lambda^{4}}{u^{2}}\right),
$$

it is recognized that the solution of $S O(4)$ theory decomposes to two solutions of independent $S U(2)$ theories. This manifestly respects the fact $S O(4) \sim S U(2) \times S U(2)$.

We can also rewrite the Picard-Fuchs equations of the theory $\mathcal{L}_{i} \Pi=0(i=0,1)$ to Appell $F_{4}$ system where $\Pi=\oint \lambda$, and $\mathcal{L}_{i}$ are given by

$$
\begin{aligned}
& \mathcal{L}_{0}=\partial_{v}+2 \partial_{u}^{2}+2 v \partial_{v}^{2} \\
& \mathcal{L}_{1}=-4\left(\Lambda^{4}-u^{2}\right) \partial_{u}^{2}+16 u v \partial_{u} \partial_{v}+16 v^{2} \partial_{v}^{2}+8 v \partial_{v}+1
\end{aligned}
$$


Changing variables as $x=-4 v / u^{2}, y=\Lambda^{4} / u^{2}$ and taking some linear combinations, we can see that the Picard-Fuchs equations reduce to Appell $F_{4}$ system $\tilde{\mathcal{L}}_{i} H=0$ where $H=u^{-\frac{1}{2}} \Pi$ and $\tilde{\mathcal{L}}_{i}$ are given by

$$
\begin{aligned}
& \tilde{\mathcal{L}}_{0}=x(1-x) \partial_{x}^{2}-2 x y \partial_{x} \partial_{y}-y^{2} \partial_{y}^{2}+\left(\frac{1}{2}-x\right) \partial_{x}-y \partial_{y}+\frac{1}{16} \\
& \tilde{\mathcal{L}}_{1}=-x^{2} \partial_{x}^{2}-2 x y \partial_{x} \partial_{y}+y(1-y) \partial_{y}^{2}-x \partial_{x}+(1-y) \partial_{y}+\frac{1}{16}
\end{aligned}
$$

of the Picard-Fuchs equations. Let us see how the group property $S O(4) \sim S U(2) \times S U(2)$ can be realized from the point of view of the Picard-Fuchs equations. In this case, we choose the variables as $z=\Lambda^{4} /(u+\sqrt{4 v})^{2}$ and $w=\Lambda^{4} /(u-\sqrt{4 v})^{2}$ so that the PicardFuchs equations defined by (4.11) and (4.12) can be combined to following two differential equations. One is $\partial_{z} \partial_{w} \Pi=0$, which means that $\Pi$ can be decomposed to the function $f(z)$ and $g(w)$ separately. By setting $f_{0}(z)=z^{1 / 4} f(z)$ and $g_{0}(w)=w^{1 / 4} g(w)$, the second equation can be written as

$$
\begin{aligned}
z^{-\frac{1}{4}}\left((1-z) z \partial_{z}^{2}\right. & \left.+(1-z) \partial_{z}+\frac{1}{16}\right) f_{0}(z) \\
& +w^{-\frac{1}{4}}\left((1-w) w \partial_{w}^{2}+(1-w) \partial_{w}+\frac{1}{16}\right) g_{0}(w)=0 .
\end{aligned}
$$

Notice that famous Picard-Fuchs equation for $\Pi=\oint \lambda$ in the case of $S U(2)$ without matters [1], can be transformed by using the variable $z=\Lambda^{4} / u^{2}$ and setting $\Pi=u^{1 / 2} \Pi_{0}$ as follows

$$
\left(4\left(u^{2}-\Lambda^{4}\right) \partial_{u}^{2}+1\right) \Pi=0 \longrightarrow\left((1-z) z \partial_{z}^{2}+(1-z) \partial_{z}+\frac{1}{16}\right) \Pi_{0}=0,
$$

which is just the hypergeometric differential equation. Differential equation (4.15) and (4.16) manifestly show that the result of $S O(4)$ should be represented by some combinations of the solutions of two independent $S U(2)$ theories.

\section{$5 \quad$ Integrable models of type $C_{2}$}

In this section, we deal with the theory with respect to the integrable system $C_{2}$. The spectral curve of this system is given by 15

$$
f=z+\frac{\mu}{z}+W(x)=0, \quad W(x)=x^{4}-u x^{2}+v .
$$


Setting the variable $y=z+W(x) / 2$ and $\mu \propto \Lambda^{8}$, we can get the hyperelliptic curve of corresponding Seiberg-Witten model in the following form:

$$
y^{2}=W(x)^{2}-\Lambda^{8}
$$

However, looking at the simple singularity part $W(x)$ which corresponds to classical singularity of gauge theory, and counting the dimension of $\mu$ which relate to the power of $\Lambda$ of instanton corrections, the theory constructed from this curve does not correspond to any known gauge theory. This theory does not seem to have any lagrangian description, in other words, it may describe the purely strong coupling theory. Since our interest is the connection between Seiberg-Witten theories with rank two and hypergeometric series, it is worth seeing how this theory can be represented by hypergeometric series. Meromorphic differential $\lambda$ is given in the weak coupling region by

$$
\begin{aligned}
\lambda & =x W^{\prime}(x) \frac{d x}{y}=\frac{x W^{\prime}(x) d x}{W(x)} \sum_{n=0}^{\infty} \frac{\Gamma\left(n+\frac{1}{2}\right)}{\Gamma\left(\frac{1}{2}\right) n !}\left(\frac{\Lambda^{8}}{W(x)^{2}}\right)^{n} \\
& =d x \int \frac{d s}{2 \pi i} \frac{\Gamma\left(s+\frac{1}{2}\right) \Gamma(-s)(-1)^{s}}{\Gamma\left(\frac{1}{2}\right) 2 s}\left(\frac{\Lambda^{8}}{W(x)^{2}}\right)^{s} .
\end{aligned}
$$

Since $W(x)$ is not varied from the $B_{2}$ and $C_{2}$ theory, we can evaluate in a same way as before. The result can be written as

$$
\begin{aligned}
a_{1}= & u^{\frac{1}{2}} \sum_{m, n} \frac{\Gamma\left(\frac{1}{2}\right) \Gamma\left(4 n+2 m-\frac{1}{2}\right)}{\Gamma\left(-\frac{1}{2}\right) \Gamma\left(2 n+m+\frac{1}{2}\right) n ! n ! m !}\left(\frac{\Lambda^{8}}{4 u^{4}}\right)^{n}\left(-\frac{4 v}{u^{2}}\right)^{m}, \\
a_{2}= & \sqrt{\frac{v}{u}} \sum_{m, n} \frac{\Gamma\left(2 n-m-\frac{1}{2}\right) \Gamma\left(m+\frac{1}{4}\right) \Gamma\left(m+\frac{3}{4}\right)}{\Gamma\left(-\frac{1}{2}\right) \Gamma\left(\frac{1}{4}\right) \Gamma\left(\frac{3}{4}\right) n ! n ! m !}\left(\frac{\Lambda^{8}}{4 v^{2}}\right)^{n}\left(-\frac{4 v}{u^{2}}\right)^{m} \\
= & \sqrt{\frac{v}{u}} H_{7}\left(-\frac{1}{2}, \frac{1}{4}, \frac{3}{4}, 1 ; \frac{\Lambda^{8}}{4 v^{2}} ;-\frac{4 v}{u^{2}}\right), \\
a_{D}^{1}= & \frac{u^{\frac{1}{2}}}{2 \pi i} \sum_{m, n} \frac{\Gamma\left(\frac{1}{2}\right) \Gamma\left(4 n+2 m-\frac{1}{2}\right)}{\Gamma\left(-\frac{1}{2}\right) \Gamma\left(2 n+m+\frac{1}{2}\right) n ! n ! m !}\left(\frac{\Lambda^{8}}{4 u^{4}}\right)^{n}\left(-\frac{4 v}{u^{2}}\right)^{m} \\
& \times\left[-2 \psi(n+1)+4 \psi\left(4 n+2 m-\frac{1}{2}\right)-2 \psi\left(2 n+m+\frac{1}{2}\right)+\log \left(\frac{\Lambda^{8}}{4 u^{4}}\right)\right], \\
a_{D}^{2}= & \frac{1}{2 \pi i} \sqrt{\frac{v}{u}} \sum_{m, n} \frac{\Gamma\left(2 n-m-\frac{1}{2}\right) \Gamma\left(m+\frac{1}{4}\right) \Gamma\left(m+\frac{3}{4}\right)}{\Gamma\left(-\frac{1}{2}\right) \Gamma\left(\frac{1}{4}\right) \Gamma\left(\frac{3}{4}\right) n ! n ! m !}\left(\frac{\Lambda^{8}}{4 v^{2}}\right)^{n}\left(-\frac{4 v}{u^{2}}\right)^{m} \\
& \times\left[-2 \psi(n+1)+2 \psi\left(2 n-m-\frac{1}{2}\right)+\log \left(\frac{\Lambda^{8}}{4 v^{2}}\right)\right],
\end{aligned}
$$

where the Appell function $H_{7}$ is defined as [39]

$$
H_{7}(a, b, c, d ; x ; y)=\sum_{m, n} \frac{(a)_{2 n-m}(b)_{m}(c)_{m}}{(d)_{n} n ! m !} x^{n} y^{m} \text {. }
$$


Certainly it is easy to see the analytic property of this function.

Let us see how these solutions can satisfy the Picard-Fuchs equation. Picard-Fuchs equation for $\Pi=\oint \lambda$ is given by

$$
\begin{aligned}
& \mathcal{L}_{0} \Pi=\left(4 \partial_{u}^{2}+2 u \partial_{u} \partial_{v}+\partial_{v}\right) \Pi=0 \\
& \mathcal{L}_{2} \Pi=\left(\left(-4 u^{2}+32 v\right) \partial_{u}^{2}+16\left(\Lambda^{8}-v^{2}\right) \partial_{v}^{2}-1\right) \Pi=0 .
\end{aligned}
$$

By direct change of the variable to $x=\Lambda^{8} / 4 v^{2}$ and $y=-4 v / u^{2}$, and some linear combinations, we see that these equations become Appell system $H_{7}$ for $\Pi_{0}=\sqrt{u / v} \Pi$ as follows

$$
\begin{aligned}
& \left(y(1+y) \partial_{y}^{2}-2 x \partial_{x} \partial_{y}+\left(\frac{3}{2}+2 y\right) \partial_{y}+\frac{3}{16}\right) \Pi_{0}=0 \\
& \left(-(4 x-1) x \partial_{x}^{2}-y^{2} \partial_{y}^{2}+4 x y \partial_{x} \partial_{y}+(-4 x+1) \partial_{x}-y \partial_{y}+\frac{1}{4}\right) \Pi_{0}=0 .
\end{aligned}
$$

Thus we see that $\Pi_{0}$ is just a Appell function $H_{7}$ and dual Higgs pairs can be represented by independent solutions of this system, which are just the solution we have obtained in this section.

It is also possible to consider other parameterization of the curve as

$$
\tilde{u}=u, \quad \tilde{v}=-v+\frac{u^{2}}{8} .
$$

In this case, solutions are obtained from previous solutions by changing variables and analytic continuations. However since the result of this manipulation depend on the choice of the branch of the logarithmic function, we instead take a different parameterization of the curve initially and perform the evaluation. $W(x)$ now can be written as

$$
\widetilde{W}(x)=\left(x^{2}-\frac{1}{2}\left(1+\frac{1}{\sqrt{2}}\right) \tilde{u}\right)\left(x^{2}-\frac{1}{2}\left(1-\frac{1}{\sqrt{2}}\right) \tilde{u}\right)-\tilde{v},
$$

therefore we regard $a_{1}$ as the contribution from $x^{2} \sim \frac{1}{2}\left(1+\frac{1}{\sqrt{2}}\right) \tilde{u}$ and $a_{2}$ from $x^{2} \sim$ $\frac{1}{2}\left(1-\frac{1}{\sqrt{2}}\right) \tilde{u}$. Expanding $\lambda$ suitably and integrating from $x=0$ to $x=\sqrt{\frac{1}{2}\left(1 \pm \frac{1}{\sqrt{2}}\right) \tilde{u}}$ with appropriate regularization, we can give the result by using $F_{4}$ function. For example $a_{1}$ can be written as

$$
\begin{aligned}
a_{1}=\frac{\sqrt{\left(1+\frac{1}{\sqrt{2}}\right) \tilde{u}}}{\sqrt{2}} F_{4}( & \left.-\frac{1}{8}, \frac{1}{8}, 1, \frac{1}{2} ; \frac{64 \Lambda^{8}}{\tilde{u}^{4}}, \frac{64 \tilde{v}^{2}}{\tilde{u}^{4}}\right) \\
& +\frac{\sqrt{2-\sqrt{2}} \tilde{v}}{\sqrt{2} \tilde{u}^{\frac{3}{2}}} F_{4}\left(\frac{3}{8}, \frac{5}{8}, 1, \frac{3}{2} ; \frac{64 \Lambda^{8}}{\tilde{u}^{4}}, \frac{64 \tilde{v}^{2}}{\tilde{u}^{4}}\right) .
\end{aligned}
$$


It is possible to obtain $a_{2}$ and $a_{D}^{1}, a_{D}^{2}$ as linear combinations of $F_{4}$ functions similarly. We can also check these solutions can satisfy the Picard-Fuchs equation directly by choosing variables as in (5.12).

There is another way to express dual Higgs pairs by Appell functions. If we consider the branch for large $v$ in the original parameterization, the result can be expressed in terms of $H_{4}$ function [39] by similar evaluations. This situation in which dual Higgs pairs can be represented by several kinds of Appell functions in each region is analogous to the relation between the weak coupling region and around the conformal point in the case of $S U(3)$ 26.

\section{Exceptional group $G_{2}$}

In this section we deal with the theory with gauge group $G_{2}$. As was pointed out in [16, 17], since there is no reliable hyperelliptic curve for $G_{2}$ so far, we start with spectral curve for integrable systems. The spectral curve for $G_{2}^{\vee}$ is given by [15]

$$
\begin{aligned}
f & =3\left(z-\frac{\mu}{z}\right)-x^{8}+2 u x^{6}-\left[u^{2}+6\left(z+\frac{\mu}{z}\right)\right] x^{4}+\left[v+2 u\left(z+\frac{\mu}{z}\right)\right] x^{2} \\
& =3\left(z-\frac{\mu}{z}\right)^{2}+\left(z+\frac{\mu}{z}\right)\left(\frac{u}{3}-x^{2}\right) 6 x^{2}-P(x) .
\end{aligned}
$$

where

$$
P(x)=x^{2}\left[x^{2}\left(x^{2}-u\right)^{2}-v\right]
$$

Holomorphic one-form for periods is given from this spectral curve by

$$
\int \frac{d x d z}{z \cdot f}
$$

Integrating with respect to $v$ we can get meromorphic differential which produces dual Higgs pairs of corresponding Seiberg-Witten model. Expanding with respect to $1 / P(x)$, holomorphic one-form is given by

$$
\int \frac{d x d z}{z f}=d x \sum_{n, k} \frac{\Gamma(n+k+1) x^{4 k}\left(\frac{u}{3}-x^{2}\right)^{2 k}(4 \mu)^{n}}{\Gamma(n-k+1) \Gamma\left(k-n+\frac{1}{2}\right) n ! k !}\left(\frac{P(x)}{3}\right)^{-(n+k+1)} .
$$


The detailed procedure is given in Appendix B. Thus meromorphic differential $\lambda$ can be written as follows

$$
\lambda=d x \int \frac{d s}{2 \pi i} \sum_{k} \frac{\Gamma(s+k) \Gamma(-s)(-1)^{s}(4 \mu)^{s} x^{4 k}\left(\frac{u}{3}-x^{2}\right)^{2 k}}{\Gamma(s-k+1) \Gamma\left(k-s+\frac{1}{2}\right) k !}\left(\frac{P(x)}{3}\right)^{-(s+k)} .
$$

In the region $u \sim \infty$, we expand this expression with respect to $1 / u$ and deform the contour to evaluate $x$ integral for Higgs pairs explicitly. By picking up poles $s=0,1, \cdots$, the contribution from $x^{2} \sim u$ and $x^{2} \sim u / u^{2}$ to lower orders of $\mu$, which amount to $a_{1}$ and $a_{2}$ respectively, turn out to be completely match the result obtained by Ito 38]. However it is difficult to combine this expression to simple known hypergeometric functions. Therefore we choose a following parameterization $\tilde{u}, \tilde{v}$ which makes the calculation simple enough to evaluate analytically

$$
\tilde{u}=u, \quad v=\frac{4}{27} u^{3}+\tilde{v} .
$$

In this parameterization, $P(x)$ shows the classical singularity as

$$
P(x)=x^{2}\left[\left(x^{2}-\frac{\tilde{u}}{3}\right)^{2}\left(x^{2}-\frac{4 \tilde{u}}{3}\right)-\tilde{v}\right] .
$$

We are going to evaluate the contribution from $x^{2} \sim \tilde{u} / 3$ and $x^{2} \sim 4 \tilde{u} / 3$. At first we attempt to calculate the contribution from $x^{2} \sim 4 \tilde{u} / 3$, however it is still difficult to combine the result to simple form. Then we instead try to calculate $a_{1}$ from the contribution from $x^{2} \sim \tilde{u} / 3$. Since this point is a double root of $P(x)=0$, the result of the calculation consist of one independent solution only, which we call even part $a^{+}$. Expanding meromorphic differential (6.5) in terms of large $\tilde{u}$, performing the line integral from $x=-\sqrt{\tilde{u} / 3}$ to $x=\sqrt{\tilde{u} / 3}$ multiplied by $\sin 2 \pi s / \pi$, we can give the solution in the following form:

$$
a_{1}=a^{+}=u^{\frac{1}{2}} \sum_{m, n} \frac{\Gamma\left(\frac{1}{2}\right) \Gamma\left(4 m+3 n-\frac{1}{2}\right)}{\Gamma\left(-\frac{1}{2}\right) \Gamma\left(m+n+\frac{1}{2}\right)^{2} m ! m ! n !}\left(\frac{9 \mu}{\tilde{u}^{4}}\right)^{m}\left(\frac{\tilde{v}}{4 \tilde{u}^{3}}\right)^{n} .
$$

The derivation of this expression is explained in detail in Appendix B. Another polynomial solution which we call odd part $a^{-}$can be given by shifting $n$ to $n-m+1 / 2$ as

$$
a^{-}=\frac{\tilde{v}^{\frac{1}{2}}}{\tilde{u}} \sum_{m, n} \frac{\Gamma\left(\frac{3}{2}\right) \Gamma(m+3 n+1)}{\Gamma\left(n-m+\frac{3}{2}\right) m ! m ! n ! n !}\left(\frac{36 \mu}{\tilde{u} \tilde{v}}\right)^{m}\left(\frac{\tilde{v}}{4 \tilde{u}^{3}}\right)^{n},
$$

which is because of the fact that this replacement does not change the recursion relation satisfied by the coefficients. The solution (6.8) and (6.9) both have forms of hypergeometric series with order three in terms of one variable $\tilde{v} / 4 \tilde{u}^{3}$. Thus we see that in the 
case of $G_{2}$ dual Higgs pairs are not within the Appell system. If we can evaluate the contribution from $x^{2} \sim 4 \sqrt{\tilde{u} / 3}$, we would see that $a_{2}$ can be written by a linear combination of $a^{+}$and $a^{-}$. Similarly we can give the logarithmic solutions $a_{D}^{+}$and $a_{D}^{-}$. An order three hypergeometric function satisfies suitable order three differential equation by definition. Odd part solution $a^{-}$is of order 2 in terms of $36 \mu / \tilde{u} \tilde{v}$, and of order 3 in terms of $\tilde{v} / 4 \tilde{u}^{3}$, therefore two more independent solutions seem to be needed. However looking at the form of these solutions, we recognize that the number of independent solution of this system is four including logarithmic solutions. This is implied by the fact that the Picard-Fuchs equations of this theory are order two differential equations [38.

In the case of $G_{2}$, we have succeeded in evaluating dual Higgs pairs explicitly by means of parameterization (6.6) only, and have not been able to sum up to the simple form by use of other parameterizations so far. Thus we recognize that $G_{2}$ seems to be exceptional case compared to the classical gauge group where we can evaluate in various parameterizations.

Let us check whether these solutions can satisfy the Picard-Fuchs equations. PicardFuchs equations for $\Pi=\oint \lambda$ are given by $\mathcal{L}_{i} \Pi=0(i=1,2)$ where 38

$$
\begin{gathered}
\mathcal{L}_{1}=\left(\frac{8 u^{3} v}{3}-36 v^{2}+960 u^{2} \mu\right) \partial_{v}^{2}+\left(\frac{8 u^{4}}{3}-24 u v+2304 \mu\right) \partial_{u} \partial_{v}+\left(4 u^{3}-24 v\right) \partial_{v}-1 \\
\mathcal{L}_{2}=\frac{2\left(720 u^{2} \mu+2 u^{3} v-27 v^{2}\right)}{-u v+24 \mu} \partial_{u}^{2}+\frac{4\left(256 u^{4} \mu-3 u^{2} v^{2}-720 u v \mu+13824 \mu^{2}\right)}{-u v+24 \mu} \partial_{u} \partial_{v} \\
-\frac{6\left(-256 u^{3} \mu+96 v \mu+5 u v^{2}\right)}{-u v+24 \mu} \partial_{v}-1 .
\end{gathered}
$$

By direct change of the variable $x=36 \mu / \tilde{u} \tilde{v}, y=\tilde{v} / 4 \tilde{u}^{3}$, and applying (6.9) to this system, we can check directly that the solution $a^{-}$satisfies these Picard-Fuchs equations order by order. Also we can show that the recursion relations with respect to these equations can be satisfied by coefficient $c(m, n)$ in arbitrary integer $m, n$ where we set $a^{-}=\tilde{v}^{\frac{1}{2}} / \tilde{u} \sum_{m, n} c(m, n) x^{m} y^{n}$. Therefore we regard $a^{+}, a^{-}$and their corresponding logarithmic solutions as four independent solutions of the Picard-Fuchs equations. However this occurs nontrivially in a sense that we could not succeed to combine $\mathcal{L}_{i}$ to differential equations $\tilde{\mathcal{L}}_{i} \Pi_{0}=0(i=1,2)$ where

$$
\begin{aligned}
\tilde{\mathcal{L}}_{1}= & (1+x) x \partial_{x}^{2}+2 x y \partial_{x} \partial_{y}-3 y^{2} \partial_{y}^{2}+\left(1+\frac{3 x}{2}\right) \partial_{x}-\frac{11}{2} y \partial_{y}-\frac{1}{2}, \\
\tilde{\mathcal{L}}_{2}= & -z^{3} \partial_{x}^{3}-\left(x y+27 x y^{2}\right) \partial_{x} \partial_{y}^{2}-9 x^{2} y \partial_{x}^{2} \partial_{y}+(1-27 y) y^{2} \partial_{y}^{3}-9 x^{2} \partial_{x}^{2} \\
& +\left(\frac{7 y}{2}-162 y^{2}\right) \partial_{y}^{2}-(x+72 x y) \partial_{x} \partial_{y}-18 x \partial_{x}+\left(\frac{3}{2}-114 y\right) \partial_{y}-6,
\end{aligned}
$$


which should be simply satisfied by $\Pi_{0}=\tilde{u} / \tilde{v}^{\frac{1}{2}} a^{-}$by definition. This is analogous to the case of Calabi-Yau 43] where the system has the redundancy which appears as the factorization of some differential operators. This feature decreases the order of differential equations satisfied by the solution, to give the essential Picard-Fuchs equations. Unfortunately we have not succeed in finding suitable parameterization which makes us possible to do in practice so far.

\section{Conclusion}

We have showed how dual Higgs pairs for Seiberg-Witten theories with rank two gauge groups can be represented by hypergeometric series in the weak coupling region both by using explicit evaluations and by the Picard-Fuchs equations. In the case of classical gauge groups, they are written by Appell functions which is natural extensions of the hypergeometric function in two variables. In the case of the exceptional group, however, we need to extend one more order, that is, they can be expressed by order three hypergeometric series in terms of one of two variables. Compared to classical groups this is realized nontrivially in a following sense that equations which should be satisfied by these solutions, look like independent from the Picard-Fuchs equations of the theory, although these solutions can satisfy the Picard-Fuchs equations.

Explicit evaluations are not in principle suffered from the complexity coming from gauge group being higher rank. However analytic property of each variable of the resulting expressions are not always apparent [26]. In order to clarify this we need more information about generalized hypergeometric functions with multi-variable. We hope that this makes us possible to analyze the behavior in the strong coupling region of Seiberg-Witten type theories with arbitrary gauge groups, even within exceptional groups, in future.

\section{A Explicit evaluations in the case of classical group}

Consider the curve of following form:

$$
y^{2}=W(x)^{2}-x^{k} \Lambda^{d}
$$


Meromorphic differential is given by

$$
\lambda=\frac{x W^{\prime}(x)-k / 2 W(x)}{y} d x .
$$

Dual Higgs pairs $a_{i}, a_{D}^{i}(i=1,2)$ are given by integration along independent suitable homology cycle $\alpha_{i}, \beta_{i}$ cycle respectively. In the weak coupling region $\Lambda \sim 0$, we expand $\lambda$ with $1 / W(x)^{2}$

$$
\lambda=d x\left(x W^{\prime}(x)-\frac{k}{2} W(x)\right) \sum_{n} \frac{\Gamma\left(n+\frac{1}{2}\right)}{\Gamma\left(\frac{1}{2}\right) n !}\left(\frac{\Lambda^{d} x^{k}}{W(x)^{2}}\right)^{n} .
$$

Introducing $s$ integral in place of summation in terms of $n$, and integrating by parts, we see that dual Higgs pairs consist of the contribution from classical root $W(x)=0$ of the curve as

$$
\lambda=d x \int_{-i \infty}^{i \infty} \frac{d s}{2 \pi i} \frac{\Gamma\left(s+\frac{1}{2}\right) \Gamma(-s)(-1)^{s}}{\Gamma\left(\frac{1}{2}\right) 2 s}\left(\frac{\Lambda^{d} x^{k}}{W(x)^{2}}\right)^{s},
$$

where we take poles at $s=0,1,2, \cdots$. In the case of $C_{2}(S p(4))$, we instead start with holomorphic one-form with respect to hyperelliptic curve $y^{2}=x^{4} W(x)^{2}-\Lambda^{6} x^{2} W(x)$, or corresponding following form obtained from spectral curve $f=\left(z-\frac{\mu}{z}\right)^{2}+x^{2} W(x)=0$ as

$$
\int \frac{d x d z}{z \cdot f}
$$

Analyzing the residue of $z$ integral we obtain same holomorphic one-form. Integrating with respect to $v$ and expanding with $1 / W(x)$, we see that $\lambda$ can be written in a similar form as (A.4).

In the weak coupling region, since each root of hyperelliptic curve reduce to the classical root of $W(x)$, we can deform the contour for $\alpha_{i}$ and $\beta_{i}$ cycles appropriate way to enclose these roots. $a_{i}$ and $a_{D}^{i}$ are obtained by evaluating the contribution from these points by picking up suitable poles with respect to corresponding cycles, so that this reduction gives correct asymptotic behaviors in the weak coupling region.

In the case of $S U(3)$ where $W(x)=x^{3}-u x-v$, in the region $u \sim \infty, \alpha_{i}(i=1,2,3)$ enclose point $x \sim \sqrt{u},-\sqrt{u},-v / u$ respectively. Contour integral along $\alpha_{i}$ cycle can be evaluated by using the line integral with suitable regularization to produce the polynomial solution. This is possible to multiply $\sin 2 s \pi / \pi$ to the expression and perform the line integral from one root to another root of $W(x)$ and evaluate simple pole of $s$ integral. For example, the solution which consist of the difference between the contribution from 
$x \sim \sqrt{u}$ and from $x \sim-v / u \sim 0$, which we call $a_{1}$, is obtained by expanding $\lambda$ multiplied by $\sin 2 s \pi / \pi$ with large $u$ and by integrating from $x=0$ to $x=\sqrt{u}$ as [26, 37]

$$
\begin{aligned}
a_{1}=\int_{0}^{\sqrt{u}} d x \int & \frac{d s}{2 \pi i} \frac{\sin 2 \pi s}{\pi} \sum_{m} \frac{\Gamma\left(s+\frac{1}{2}\right) \Gamma(-s)(-1)^{s} \Gamma(2 s+m)}{\Gamma\left(\frac{1}{2}\right) \Gamma(2 s+1) m !}\left(\Lambda^{6}\right)^{s} v^{m} \\
& \times x^{-2 s-m}\left(x^{2}-u\right)^{-2 s-m}
\end{aligned}
$$

Performing line integral by changing variable as $x^{2}=u t$ and evaluating simple poles of resulting expression, we can give the expression (2.7) for $a_{1}$. To get corresponding $a_{D}^{1}$ which is obtained by integration along $\beta_{1}$, we integrate $\lambda$ similarly without multiplied by $\sin 2 \pi s$ and evaluate double poles and subtract $a_{1} / 2$ [26, 37]. The result can be written

as (2.8). Replacing $\sqrt{u}$ to $-\sqrt{u}$, we can obtain expression $a_{2}$, $a_{D}^{2}$ which contain the difference between the contribution from $x \sim-\sqrt{u}$ and from $x \sim-v / u$.

In $S O(5), S O(4)$ and the theory based on integrable model $C_{2}$ case, we use $W(x)=$ $x^{4}-u x^{2}+v$. The contribution comes from $x^{2} \sim u$ and $x^{2} \sim v / u$. To evaluate the former contribution, we expand $\lambda$ with $1 /\left(x^{4}-u x^{2}\right)$ and integrate in a similar way as $S U(3)$ case. To calculate latter contribution, we expand with $1 /\left(u x^{2}-v\right)$ and integrate from $x=0$ to $x=\sqrt{v / u}$, and evaluate poles regulated suitably as before. In this way we can derive the expression for $a_{i}, a_{D}^{i}$ of the theory with the classical gauge group.

\section{B Explicit evaluations in the case of exceptional group}

In the case of $G_{2}$, the spectral curve of the integrable system $G_{2}^{\vee}$ is given by (6.3). In the weak coupling region, we expand (6.1) with respect to $1 / P(x)$

$$
\begin{aligned}
& \int \frac{d x d z}{2 \pi i z f}= \frac{d x}{2 \pi i} \int \frac{d z}{z} \sum_{n}\left[\left(z-\frac{\mu}{z}\right)^{2}+2 x^{2}\left(z+\frac{\mu}{z}\right)\left(\frac{u}{3}-x^{2}\right)\right]^{n}\left(\frac{P(x)}{3}\right)^{-n-1} \\
&=\frac{d x}{2 \pi i} \int \frac{d z}{z} \sum_{n, k} \frac{n !}{k !(n-k) !}\left(z-\frac{\mu}{z}\right)^{2(n-k)}\left(z+\frac{\mu}{z}\right)^{k} \\
& \times\left[2 x^{2}\left(\frac{u}{3}-x^{2}\right)\right]^{k}\left(\frac{P(x)}{3}\right)^{-n-1}
\end{aligned}
$$

Expanding both braces of integrant, we evaluate the residue at $z=0$

$$
\frac{1}{2 \pi i} \int \frac{d z}{z}\left(z-\frac{\mu}{z}\right)^{2(n-k)}\left(z+\frac{\mu}{z}\right)^{k}
$$




$$
\begin{aligned}
& =\sum_{i} \frac{(2(n-2 k)) !(2 k) ! \mu^{n-k}(-1)^{i}}{\Gamma(2 n-4 k-i+1) \Gamma(3 k-n+i) \Gamma(n-k-i+1) i !} \\
& =\frac{(2 k) ! \mu^{n-k}}{\Gamma(3 k-n+1) \Gamma(n-k+1)} F(4 k-2 n, k-n, 3 k-n+1 ;-1) .
\end{aligned}
$$

Using the value of the hypergeometric function 39]

$$
F(4 k-2 n, k-n, 3 k-n+1,-1)=\frac{2^{-4 k+2 n} \Gamma(3 k-n+1) \Gamma\left(\frac{1}{2}\right)}{\Gamma(k+1) \Gamma\left(2 k-n+\frac{1}{2}\right)},
$$

we can get expression (6.4) which corresponds to holomorphic one-form. Thus the meromorphic differential is given by

$$
\lambda=d x \int \frac{d s}{2 \pi i} \sum_{k} \frac{\Gamma(s+k) \Gamma(-s)(-1)^{s}(4 \mu)^{s} x^{4 k}\left(\frac{u}{3}-x^{2}\right)^{2 k}}{\Gamma(s-k+1) \Gamma\left(k-s+\frac{1}{2}\right) k !}\left(\frac{P(x)}{3}\right)^{-(s+k)},
$$

where we introduce $s$ integral $(s=0,1,2, \cdots)$ as before.

We take different parameterization convenient for evaluation of the integral as

$$
P(x)=x^{2}\left[\left(x^{2}-\frac{\tilde{u}}{3}\right)^{2}\left(x^{2}-\frac{4 \tilde{u}}{3}\right)-\tilde{v}\right] .
$$

We are going to evaluate the contribution from $x^{2} \sim u / 3$. To get the polynomial solution $a^{+}$, we calculate

$$
a^{+}=\int_{-\sqrt{\frac{u}{3}}}^{\sqrt{\frac{\tilde{u}}{3}}} \lambda \frac{\sin 2 \pi(s+k)}{\pi}
$$

where we expand $\lambda$ with $1 /\left(x^{2}-u / 3\right)$. In order to perform $x$ integral we use integral representation for the hypergeometric function

$$
F(a, b, c ; z)=\frac{\Gamma(c)}{\Gamma(b) \Gamma(c-b)} \int_{0}^{1} d t t^{b-1}(1-t)^{c-b-1}(1-z t)^{-a},
$$

so that we give the following expression

$$
\begin{aligned}
a^{+}=\tilde{u}^{\frac{1}{2}} \int \frac{d s}{2 \pi i} \sum_{k, m} \frac{\sin 2 \pi(s+k)}{\pi} \frac{\Gamma(-s)(-1)^{s} \Gamma(s+k+m)}{\Gamma(s-k+1) \Gamma(k+1) \Gamma(m+1)} \\
\times \frac{\Gamma\left(\frac{3}{2}\right) \Gamma(-2 s-2 m+1)}{\Gamma\left(-3 s+k-2 m+\frac{3}{2}\right)}\left(\frac{4 \mu}{\tilde{u}^{4}}\right)^{s}\left(\frac{\tilde{v}}{\tilde{u}^{3}}\right)^{m} \frac{3^{5 s+k+3 m}}{(-4)^{s+k+m}} \\
\quad \times F\left(s+k+m,-s+k+\frac{1}{2},-3 s+k-2 m+\frac{3}{2} ; \frac{1}{4}\right) .
\end{aligned}
$$


We want to modify this expression to simple form. First of all we evaluate the value of the hypergeometric function by using analytic continuation formula

$$
\begin{aligned}
F(a, b, c ; z)=(1-z)^{-a} & \frac{\Gamma(c) \Gamma(b-a)}{\Gamma(b) \Gamma(c-a)} F\left(a, c-b, a-b+1 ; \frac{1}{1-z}\right) \\
& +(1-z)^{-b} \frac{\Gamma(c) \Gamma(a-b)}{\Gamma(a) \Gamma(c-b)} F\left(b, c-a, b-a+1 ; \frac{1}{1-z}\right),
\end{aligned}
$$

where $a=s+k+m, b=-s+k+1 / 2, c=-3 s+k-2 m+3 / 2$ and $z=1 / 4$. Notice that in this case there is no contribution from second term because poles with respect to $s$ disappear after this manipulation. Then we have

$$
\begin{gathered}
a^{+}=\tilde{u}^{\frac{1}{2}} \int \frac{d s}{2 \pi i} \sum_{k, m} \frac{\sin 2 \pi(s+k)}{\pi} \frac{\Gamma(-s)(-1)^{s} \Gamma(s+k+m) 3^{4 s+2 m}(-1)^{s+k+m}}{\Gamma(s-k+1) \Gamma(k+1) \Gamma\left(k-s+\frac{1}{2}\right) \Gamma(m+1)} \\
\times \frac{\Gamma\left(\frac{3}{2}\right) \Gamma(-2 s-2 m+1) \Gamma\left(-2 s-m+\frac{1}{2}\right)}{\Gamma\left(-4 s-3 m+\frac{3}{2}\right)}\left(\frac{4 \mu}{\tilde{u}^{4}}\right)^{s}\left(\frac{\tilde{v}}{\tilde{u}^{3}}\right)^{m} \\
\times F\left(s+k+m,-2 s-2 m+1,2 s+m+\frac{1}{2} ; \frac{4}{3}\right) .
\end{gathered}
$$

Next we intend to sum up with respect to $k$. Getting hypergeometric function back to the form of summation in term of $l$, and combining $k$ summation to hypergeometric function with unit value which is given by 39

$$
F(a, b, c ; 1)=\frac{\Gamma(c) \Gamma(c-a-b)}{\Gamma(c-a) \Gamma(c-b)},
$$

we give the following expression by rewriting again resulting $l$ summation to hypergeometric function with value $4 / 3$

$$
\begin{aligned}
a^{+}=\tilde{u}^{\frac{1}{2}} \int \frac{d s}{2 \pi i} \sum_{m} & \frac{\Gamma\left(\frac{1}{2}\right) \Gamma(-s)(-1)^{s} \Gamma(s+m) \Gamma\left(4 s+3 m-\frac{1}{2}\right) 3^{4 s+2 m}}{\Gamma\left(-\frac{1}{2}\right) \Gamma(s+1) \Gamma(m+1) \Gamma(2 s+2 m) \Gamma\left(\frac{1}{2}+s+m\right)} \\
& \times\left(\frac{4 \mu}{\tilde{u}^{4}}\right)^{s}\left(\frac{\tilde{v}}{\tilde{u}^{3}}\right)^{m} F\left(1-2 s-2 m, s+m ; \frac{1}{2}+s+m ; \frac{4}{3}\right) .
\end{aligned}
$$

Finally in order to evaluate the value of the hypergeometric function, we try to use next formula for arbitrary integer $n$

$$
F\left(-2 n+1, n ; \frac{1}{2}+n ; \frac{4}{3}\right)=\frac{1}{3^{2 n}} .
$$

This formula is not sited elsewhere, however we can verify directly by substituting small integer $n$, and for large $n$ by using mathematica. Using this formula, we can get $a^{+}$in the form:

$$
a^{+}=u^{\frac{1}{2}} \sum_{m, n} \frac{\Gamma\left(\frac{1}{2}\right)^{2} \Gamma\left(4 m+3 n-\frac{1}{2}\right)}{\Gamma\left(-\frac{1}{2}\right) \Gamma\left(m+n+\frac{1}{2}\right)^{2} m ! m ! n !}\left(\frac{9 \mu}{u^{4}}\right)^{m}\left(\frac{\tilde{u}}{4 u^{3}}\right)^{n} .
$$




\section{References}

[1] N.Seiberg and E.Witten, Nucl. Phys. B426 19 (1994); Nucl. Phys. B431 484 (1994).

[2] P.C.Argyres, M.R.Douglas, Nucl. Phys. B448 93 (1996), hep-th/9505062.

[3] P.C.Argyres, R.N.Plesser, N.Seiberg and E.Witten, Nucl. Phys. B461 71 (1996), hep-th/9511154.

[4] T.Eguchi, K.Hori, K.Ito and S.K.Yang, Nucl. Phys. B471 430 (1996), hepth/9603002.

[5] A.Klemm, W.Lerche, S.Theisen and S.Yankielowics, Phys. Lett. B344 196 (1995); C.P.Argyres and A.Farragi, Phys. Rev. Lett. 743931 (1995).

[6] P.C.Argyres, R.Plesser and A.Shapere, Phys. Rev. Lett. 751699 (1995), hepth/9505100;

J.Minahan and D.Nemeschansky, Nucl. Phys. B464 3 (1996), hep-th/9507032.

[7] A.Hanany and Y.Oz, Nucl. Phys. B452 283 (1995), hep-th/9505073

[8] U.Danielson and B.Sundborg, Phys. Lett. B358 273 (1995), hep-th/9504102; Phys. Lett. B370 83 (1996), hep-th/11180.

A.Brandhuber and K.Landsteiner, Phys. Lett. B358 73 (1995), hep-th/ 9507008.

R.Donagi and E.Witten, Nucl. Phys. B460 299 (1996), hep-th/9510101.

[9] A.Hanany, Nucl. Phys. B466 85 (1996), hep-th/9509176.

[10] P.C.Argyres and A.Shapere, Nucl. Phys. B461 437 (1996), hep-th/9509175.

[11] A.Ceresole, R.D'Auria and S. Ferrara, Phys. Lett. B339 71 (1994).

S.Ryang, Phys. Lett. B365 113 (1996), hep-th/9508163.

[12] A.Klemm, W.Lerche and S.Theisen, Int. Jour. Mod. Phys. A11 1929 (1996), hepth/9505015.

[13] A.Brandhuber and S.Stieberger, "Periods, Coupling Constants and Modular Functions in N=2 SU(2) SYM with Massive Matter", hep-th/9609130.

[14] T.Nakatsu and K.Takasaki, Mod. Phys. Lett. All 157 (1996), hep-th/9509162.

E.Martinec, Phys. Lett. B367 91 (1996), hep-th/9510204.

H.Itoyama and A.Morozov, Nucl. Phys. B477 855 (1996), hep-th/9511126.

[15] E.Martinec and N.Warner, Nucl. Phys. B459 97 (1996), hep-th/9509161.

[16] M.Alishahiha, G.Ardalan and F.Mansouri, Phys. Lett. B381 446 (1996), hepth/9512005.

K.Landsteiner, J.Pierre and S.Giddings, "On the Moduli Space of N=2 Supersymmetric $G_{2}$ Gauge Theory", hep-th/9609059. 
[17] M.R.Abolhasani, M.Alishahiha and A.M.Ghezelbash, Nucl. Phys. B480 279 (1996), hep-th/9606043.

[18] W.Lerche and N.Warner, "Exceptional SW Geometry from ALE Fibrations ", hepth/9608183.

[19] S.Kachru, A.Klemm, W.Lerche, P.Mayr and C.Vafa, Nucl. Phys. B459 537 (1996), hep-th/9508155.

[20] A.Klemm, W.Lerche, P.Mayr, C.Vafa and N.Warner, Nucl. Phys. B477 746 (1996), hep-th/9604034.

[21] W.Lerche, "Introduction to Seiberg-Witten Theory and its Stringy Origin", hepth/9611190.

[22] A.Klemm, "On the Geometry behind N=2 Supersymmetric Effective Actions in Four Dimensions", hep-th/9705131.

[23] J.H.Brodie, "Exact Solutions of Exceptional Gauge Theories from Toric Geometry", hep-th/9705068.

[24] J.M.Isidro, A.Mukherjee, J.P.Nunes and H.J.Schnitzer, "A New Derivation of the Picard-Fuchs Equations for Effective N=2 Super Yang-Mills Theories", hepth/9609110; "A Note on the Picard-Fuchs Equations for $N=2$ Seiberg-Witten Theories", hep-th/9703176.

M. Alishahiha, "On the Picard-Fuchs equations of the SW models", hep- th/9609157; "Simple Derivation of the Picard-Fuchs Equations for the Seiberg-Witten Models", hep-th/9703186.

[25] E.D'Hoker, I.Krichever and D.Phong, Nucl. Phys. B490 40 (1997), hep-th/9609079; Nucl. Phys. B489 211 (1997), hep-th/9609145

[26] T.Masuda and H.Suzuki, "On explicit evaluations around the conformal point in $N=2$ supersymmetric Yang-Mills theories", hep-th/9612240.

[27] M.Matone, Phys. Lett. B357 342 (1995), hep-th/9506102.

[28] T.Eguchi and S.K.Yang, Mod. Phys. Lett. A11 131 (1996), hep-th/9510183.

H.Itoyama and A.Morozov, "Prepotential and the Seiberg-Witten Theory", hepth/9512161.

G.Bonelli and M.Matone, Phys. Rev. Lett. 764170 (1996), hep-th/9602174; Phys. Rev. Lett. 774712 (1996), hep-th/9605090.

[29] D.Finnell and P.Pouliot, Nucl. Phys. B453 225 (1996), hep-th/9503115.

A.Yung, Nucl. Phys. B485 38 (1997), hep-th/9605096.

F.Fucito and G.Travaglini, Phys. Rev. D55 1099 (1997), hep-th/9605215.

N.Dorey, V.A.Khoze and M.Mattis, Phys. Rev. D54 2921 (1996), hep-th/9603136; 
Phys. Lett. B390 205 (1997), hep-th/9606199; Phys. Lett. B388 324 (1996), hepth/9607066; Phys. Rev. D54 7832 (1996), hep-th/9607202.

K.Ito and N.Sasakura, Phys. Lett. B382 95 (1996); hep-th/9602073; Mod. Phys. Lett. A12 205 (1997); hep-th/9609104

T.Harano and M.Sato, Nucl. Phys. B484 167 (1997), hep-th/9608060.

H.Aoyama, T.Harano, M.Sato and S.Wada, Phys. Lett. B388 331 (1996), hepth/9607076.

[30] K. Ito and N. Sasakura, Nucl. Phys. B484 141 (1997), hep-th/9608054.

[31] E.D'Hoker and D.Phong, Phys. Lett. B39794(1997), hep-th/9701055.

[32] A.Bilal and F.Ferrari, Nucl. Phys. B469 387 (1996), hep-th/9602082; Nucl. Phys. B480 589 (1996), hep-th/9605101

[33] L.Alvarez-Gaume, M.Marino and F.Zamora, Int. J. Mod. Phys. A11 4745 (1996), hep-th/9604004; Int. J. Mod. Phys. A12 975 (1997), hep-th/9606191; "Softy broken $N=2$ QCD with Massive Quark Hypermultiplets, hep-th/9702054.

[34] K.Ito and S.-K.Yang, Phys. Lett. B366 165 (1996), hep-th/9507144; "Picard-Fuchs Equations and Prepotentials in N=2 Supersymmetric QCD”, hep-th/9603073.

[35] H.Ewen, K.Forger and S.Theisen, Nucl. Phys. B485 63 (1997), hep-th/9609062.

H.Ewen and K.Forger, "Simple Calculation of Instanton Corrections in Massive $N=2 S U(3) S Y M "$, hep-th/9610049.

[36] Y.Ohta, J. Math. Phys. 376074 (1996), hep-th/9604051; J. Math. Phys. 38682 (1997), hep-th/9604059.

[37] T. Masuda and H. Suzuki, "Prepotential of $N=2$ Supersymmetric Yang-Mills Theories in the Weak Coupling Region", hep-th/9609065; "Periods and Prepotential of N=2 SU(2) Supersymmetric Yang-Mills Theory with Massive Hypermultiplets", hep-th/9609066.

[38] K.Ito, "Picard-Fuchs Equations and Prepotential in $N=2$ Supersymmetric $G_{2}$ YangMills Theory", hep-th/9703180.

[39] see for example, A.Erdélyi et al., "Higher Transcendal Functions",(McGraw-Hill, New York) Vol.1.

[40] A.Erdélyi, Proc. Roy. Soc. Edinburgh Sect. A62 378 (1948).

[41] P.Appell and M.J.Kanpé de Fériet, "Fonctions hypergeómétriques et hypersphériques. Polynomes d'Hermite." Gauthier-Villars. (1926).

[42] H.Suzuki, "Enhanced Gauge Symmetry in Three Moduli Models of Type II String and Hypergeometric Series", hep-th/9701094.

[43] S.Hosono, A.Klemm, S.Theisen and S.T.Yau, Nucl. Phys. B433 501 (1995), hepth/9406055. 